УДК 621.315 .592

\title{
Расчет влияния плотности ионного тока и температуры на кинетику накопления точечных дефектов при облучении кремния легкими ионами
}

\author{
(C) Е.В. Окулич ${ }^{1}$, В.И. Окулич ${ }^{2, \uparrow}$, Д.И. Тетельбаум ${ }^{1}$ \\ ${ }^{1}$ Нижегородский государственный университет им. Н.И. Лобачевского, \\ 603950 Нижний Новгород, Россия \\ ${ }^{2}$ Нижегородский институт управления - филиал Российской академии народного хозяйства \\ и государственной службы при президенте Российской Федерации, \\ 603950 Нижний Новгород, Россия \\ E-mail: victorokulich@mail.ru
}

(Получена 14 декабря 2017 г. Принята к печати 25 декабря 2017 г.)

Выполнены численные расчеты кинетики накопления точечных дефектов - вакансий и дивакансий при облучении кремния ионами с массами $M_{1} \leq 31$ а.е.м. и энергиями $E \leq 100$ кэВ при различных условиях облучения. Использована ранее предложенная диффузионно-коагуляционная модель, но без сделанного при ее аналитической реализации приближения „слабой диффузии“. Проанализированы основные особенности зависимостей концентраций вакансий и дивакансий от дозы, плотности ионного тока и температуры при облучении. Дана физическая интерпретация полученных результатов. Разработанный вычислительный комплекс является достаточно гибким, позволяя путем вариации входных параметров модели анализировать их влияние, а также при необходимости включать в рассмотрение дополнительные процессы.

DOI: $10.21883 /$ FTP.2018.09.46228.8720

\section{1. Введение}

Радиационное повреждение полупроводников является одной из центральных проблем ионной имплантации. Хотя эта проблема подвергалась интенсивному экспериментальному и теоретическому исследованиям, в особенности для наиболее важного для электронной техники полупроводника - кремния, она сохраняет свою актуальность, в частности, в связи с необходимостью снижения температуры имплантационного отжига при уменьшении размеров активных областей приборов (см., например, [1]).

Ранние теоретические работы по кинетике накопления дефектов при ионной имплантации базировались преимущественно на феноменологических подходах решении кинетических уравнений, описывающих поведение элементарных дефектов, генерируемых при ионном облучении (вторичное дефектообразование) [2-4]. С развитием компьютерной техники появилась возможность моделирования процессов при облучении на атомарном уровне. Использовались такие продвинутые способы, как молекулярная динамика (МД) [5] и кинетический метод Монте-Карло (КМК) [6]. Применение этих методов выявило ряд интересных особенностей трансформации кристаллической решетки, вплоть до ее полного разрушения - аморфизации. На этой основе были предложены усложненные по сравнению с прежними модели радиационного повреждения [7].

К сожалению, наиболее прямой из атомистических методов - МД, даже при использовании современной суперкомпьютерной техники, позволяет моделировать процессы лишь на протяжении очень малых времен порядка наносекунд, тогда как процессы вторичного дефектообразования характеризуются на много порядков бо́льшими временами. Кроме того, из-за потребности в больших компьютерных ресурсах МД-моделирование процессов обычно выполняют для ячеек малого размера, что не позволяет учитывать процессы взаимодействия между элементарными дефектами, разделенными большими по сравнению с размером модельной ячейки расстояниями. Такие процессы наиболее важны для легких ионов, когда „атмосфера“, состоящая из первичных дефектов - компонентов пар Френкеля (КПФ), сильно разрежена, что создает условия для их разбегания. Обычно используемые в МД-расчетах периодические граничные условия в этом случае проблематичны. Метод КМК пригоден для рассмотрения поведения КПФ при больших временах. Но в случае необходимости учета взаимодействия дефектов, образующихся в разные моменты времени в разных местах кристалла (как это имеет место при типичных плотностях ионного тока), метод КМК также является чрезмерно ресурсозатратным. В силу сказанного оба подхода чаще всего ограничиваются расчетами для избранных пар ион-атом (атомы) вещества и избранных внешних условий (температура, плотность ионного тока), что не дает полной картины зависимостей процессов накопления дефектов от этих условий.

Для приближенного рассмотрения подобных задач, на наш взгляд, можно использовать более простой и менее ресурсоемкий феноменологический подход. Такой подход был применен в работах [2,3], где пред- 
ложена простейшая модель, названная „диффузионнокоагуляционной“. В ней процессы генерации, диффузии и взаимодействия дефектов рассматриваются в одномерном приближении, при котором скорость генерации КПФ в плоскости, параллельной поверхности, считается однородной и не зависящей от времени. Такой подход в значительной степени теряет адекватность для тяжелых ионов и слишком низких температур, когда слабо выражено диффузионное „разбегание“ точечных дефектов. Очевидно, что данный подход теряет силу также при слишком малых дозах и плотностях ионного тока, когда пространственно-временное перекрытие каскадов смещения, в которых первоначально сосредоточены КПФ, слабо выражено. Другая причина, по которой данная модель теряет силу при слишком малых дозах, указана в работе [8] и связана с возможным захватом генерируемых точечных дефектов на исходные дефекты кристалла - так называемые „насыщаемые ловушки“. В этих случаях процесс накопления вторичных дефектов определяется только дозой (для определенной пары ион-атом вещества при фиксированной энергии) и не зависит от плотности ионного тока (если температура образца при изменении плотности тока поддерживается постоянной). Однако для легких ионов указанный подход в широком диапазоне условий облучения в первом приближении достаточно адекватно описывает реальную ситуацию.

В работах [2,3] при решении кинетических уравнений с целью получения аналитического решения было сделано упрощение, справедливость которого может иметь место лишь в ограниченном диапазоне условий облучения, - так называемое приближение „слабой диффузии“, позволившее исключить из уравнений диффузионный член. В настоящей работе численное решение соответствующих уравнений проводилось без использования указанного упрощения, что позволило оценить меру его справедливости.

\section{2. Модель и ее компьютерная реализация}

При ионном облучении образуются КПФ - вакансии и собственные межузельные атомы (СМА). В модели из работ [2,3] принимается, что для описания процесса накопления дефектов достаточно следить за поведением вакансий и комплексов, состоящих из двух и более вакансий, причем в отличие от одиночных вакансий эти комплексы предполагаются стабильными и неподвижными. Нами было проведено рассмотрение в предположении, что конечным продуктом объединения вакансий являются дивакансии [9] (хотя вычисления могут быть легко обобщены на случай образования комплексов из нескольких вакансий). Что касается СМА, то предполагается, что они завершают свою эволюцию на начальной стадии, в частности, образуя дефекты, служащие ненасыщаемыми ловушками для вакансий. Вакансии диффундируют и участвуют в образовании дивакансий по бимолекулярному закону. Одновременно происходит захват вакансий ненасыщаемыми ловушками.

Как уже было указано выше, поток ионов предполагается однородным по поверхности образца и непрерывным во времени. Не учитывается также возможный процесс захвата вакансий на исходные дефекты (насыщаемые ловушки) [8]. Считается, что этот процесс в основном заканчивается к моменту, когда доминирующими становятся процессы, рассматриваемые в модели из работ [2,3]. Эффект каналирования также не учитывается. В этом случае для концентраций вакансий $\left(C_{v}\right)$ и дивакансий $\left(C_{v v}\right)$ система уравнений имеет вид

$$
\left\{\begin{aligned}
\frac{\partial C_{v}(t, x)}{\partial t}= & D_{v} \frac{\partial^{2} C_{v}(t, x)}{\partial x^{2}}-K_{v v} C_{v}^{2}(t, x) \\
& -K_{v t} C_{t} C_{v}(t, x)+w G(x) \\
\frac{\partial C_{v v}(t, x)}{\partial t}= & 0.5 K_{v v} C_{v}^{2}(t, x) .
\end{aligned}\right.
$$

Здесь $w$ - плотность потока ионов $(w=j / z, j-$ плотность ионного тока, $z-$ заряд иона); $D_{v}-$ коэффициент диффузии вакансий $\left(D_{v}=D_{0} \exp \left(-E_{m} / k T\right)\right.$, где $k$ - постоянная Больцмана, $E_{m}$ - энергия миграции); $G(x)$ - функция генерации вакансий одним ионом (полное число вакансий на единицу длины нормали, генерируемых ионом на глубине $x)$. Функция $G(x)$ зависит от массы $\left(M_{1}\right)$ и начальной энергии $(E)$ иона. Величины $K_{v v}$ и $K_{v t}-$ кинетические коэффициенты реакции объединения вакансий в дивакансии и захвата вакансий на ненасыщаемые ловушки соответственно. Для них справедливы соотношения [10,11]:

$$
\begin{aligned}
& K_{v v}=8 \pi r_{i} D_{v}\left[1+\frac{r_{0}}{r_{i}} \exp \left(\frac{E_{b}}{k T}\right)\right]^{-1}, \\
& K_{v t}=4 \pi r_{i} D_{v}\left[1+\frac{r_{0}}{r_{i}} \exp \left(\frac{E_{t}}{k T}\right)\right]^{-1},
\end{aligned}
$$

где $r_{0}$ - межатомное расстояние, $r_{i}$ - радиус взаимного захвата вакансий в реакции $V+V=V_{2}, r_{t}$ - радиус захвата вакансии на ловушки, $E_{b}$ и $E_{t}-$ энергетические барьеры для реакций объединения вакансий и их захвата ловушками соответственно.

В модели не учитывается возможное изменение профиля дефектов за счет распыления (оценки показывают, что при облучении ионами с используемыми в расчетах массами $\left(M_{1} \leq 31\right.$ а.е.м. $)$, энергиями $E \geq 20$ кэВ и дозами до $\sim 10^{16} \mathrm{~cm}^{-2}$ изменение профиля дефектов за счет распыления не вносит существенного вклада). Пренебрегается также возможным влиянием естественного окисла, граница которого с кремнием может работать как геттер для дефектов [4], а также влиянием упругого и электрического полей [12].

При численном решении системы уравнений (1) толщина пластины кремния $L$ принималась равной $(5-8) \cdot R_{p}$, где $R_{p}-$ средний проецированный пробег 
ионов. Так как при этом на глубине $L$ концентрация вакансий спадает до очень низких значений по сравнению с максимальной, полученные решения можно отождествить с решениями для полубесконечной пластины. Начальное условие: $C_{v}(x, 0)=C_{v v}(x, 0)=0$. Граничное условие на границе $x=L$ взято в виде $C_{v}(L, t)=C_{v v}(L, t)=0$. С выбором граничного условия на поверхности образца $(x=0)$ дело обстоит несколько сложнее, так как в общем случае поверхность может быть частично отражающей, частично поглощающей. Расчеты проводились для двух крайних вариантов: $C_{v}(0, t)=0$ (поглощающая граница) и $\partial C_{v} / \partial t=0$ (отражающая граница).

Для функций $G(x)$ наиболее корректно было бы использовать распределения вакансий, рассчитанных по программе SRIM [13], аппроксимируя функции многочленами. Однако такая процедура оказывается слишком затратной с точки зрения машинного времени. Так, для ионов $\mathrm{B}^{+}$с энергией 100 кэВ для достаточно точного представления результатов SRIM потребовалась аппроксимация $G(x)$ полиномом 18-й степени. Гораздо менее затратной и в то же время приемлемой с учетом приближенного характера модели является аппроксимация гауссианами:

$$
G(x)=p \exp \left(-\frac{\left(x-R_{d}\right)^{2}}{2 \Delta R_{d}^{2}}\right),
$$

где $p$ - нормирующий множитель, а параметры $R_{d}$ и $\Delta R_{d}$ взяты из таблиц работы [14]. Множитель $p$ находился с использованием модифицированного соотношения Кинчина-Пиза [15-17]:

$$
\int_{0}^{L} G(x) d x=\frac{0.8 E_{n}}{2 E_{d}} .
$$

Здесь $E_{n}$ - энергия иона, затраченная на упругие столкновения [14], $E_{d}$ - пороговая энергия смещения. Расчеты показали, что выбор значения $E_{d}$ оказывает более сильное влияние на результаты, чем замена функций $G(x)$, рассчитанных по программе SRIM, на гауссианы, а также использование приближенных соотношений (4) и (5). В дальнейшем приведены результаты, полученные с использованием гауссовых функций.

Наличие развитых интегрируемых сред решения математических задач и обработки полученных данных в сочетании с высокопроизводительной вычислительной техникой позволяет проводить расчеты для широкого интервала параметров, характеризующих те или иные процессы. Нами разработан вычислительный комплекс, включающий решатель на базе пакета „Mathematica“ [18], табличный процессор Excel и плагин Mathematica for Excel, реализующий обмен данными между решателем и Excel. Рабочие книги и листы Excel используются в качестве источников входных данных и для сохранения результатов, а также для их отображения в виде таблиц и графиков.
При работе с данным комплексом в качестве входных параметров вводятся следующие величины: масса $M_{1}$ и энергия $E_{0}$ иона, доза $\Phi$, плотность ионного тока $j$, температура образца при облучении $T$, параметры диффузии вакансий и другие параметры, входящие в (2)-(5). На выходе получаем в табличном виде зависимости концентрации вакансий и дивакансий от $\Phi, j$ и $T$, которые представляются в виде концентрационных профилей.

\section{3. Результаты}

Рассчитаны профили распределения концентраций вакансий и дивакансий для разных ионов с $M_{1} \leq 31$ а.е.м. и $E \leq 100$ кэВ при различных режимах облучения в зависимости от параметров облучения. В табл. 1 указаны виды ионов и значения входящих в соотношение (4) параметров $R_{d}$ и $\Delta R_{d}$ из работы [14], а в табл. 2 даны значения других параметров, которые были использованы при расчетах. Выбор параметров производился следующим образом. Параметры $D_{0}$ и $E_{m}$ диффузии вакансий (которые считались нейтральными) заимствованы из $[19,20]$. Для $E_{d}$ использовались два значения: 22 и 15 эВ. Эти значения выбраны из интервала имеющихся в литературе данных, определенных для $\mathrm{Si}$ экспериментальным путем (см., например, [21,22]). (Современное состояние проблемы выбора параметра $E_{d}$ изложено в работе [23]. В данной статье указаны результаты расчетов при $E_{d}=22$ эВ, но все качественные закономерности остаются справедливыми и при $E_{d}=15$ эВ). Для радиусов захвата $r_{i}, r_{t}$ приняты значения, рассчитанные в [24]. Значения параметров $E_{b}, E_{t}$ и $C_{t}$ были взяты из условия наилучшего соответствия расчетов с экспериментальными данными по радиационному повреждению кремния ионами бора в зависимости от $j[25]$.

Насколько обоснован выбор указанных параметров? Так как учет диффузионного члена является важным отличием от приближенного решения, приведенного в работах [2,3], остановимся на обосновании выбора параметров диффузии вакансий. Известно, что при облучении уровень Ферми в кремнии стремится к положению, близкому к середине запрещенной зоны уже при низких (по сравнению с рассматриваемыми здесь) степенях радиационного повреждения [26]. Поэтому с учетом энергетического положения уровней вакансий $[19,20]$ при расчетах наиболее целесообразно считать вакансии нейтральными. Приведенные для них в

Таблица 1. Параметры ионов

\begin{tabular}{c|c|c|c}
\hline Сорт иона & $M_{1}$, (а.е.м.) & $R_{d}$, мкм & $\Delta R_{d}$, мкм \\
\hline $\mathrm{B}$ & 11 & 0.218 & 0.097 \\
$\mathrm{~N}$ & 14 & 0.179 & 0.085 \\
$\mathrm{Ne}$ & 20 & 0.140 & 0.070 \\
$\mathrm{Si}$ & 28 & 0.098 & 0.053 \\
$\mathrm{P}$ & 31 & 0.090 & 0.049
\end{tabular}


Таблица 2. Параметры, принятые при расчетах кинетических коэффициентов

\begin{tabular}{c|c|c|c|c|c|c|c|c}
\hline Параметр & $r_{0}, \mathrm{HM}$ & $r_{i}, \mathrm{HM}$ & $r_{t}, \mathrm{HM}$ & $E_{b},{ }^{\mathrm{B}}$ & $E_{t}$, эB & $E_{m}$, эB & $E_{d},{ }^{\mathrm{B}}$ & $C_{t}, \mathrm{~cm}^{-3}$ \\
\hline Значение & 0.234 & 0.7 & 0.7 & 0.24 & 0.06 & 0.23 & 22 & $9 \cdot 10^{17}$
\end{tabular}

работах $[19,20]$ значения диффузионных параметров мы полагаем достаточно надежными, так как они получены на основе экспериментальных данных. Параметры $E_{b}$, $E_{t}$ и $C_{t}$ можно рассматривать как подгоночные; так как они выбраны одними и теми же для разных ионов, подгонка не лишает смысла сравнение расчетных и экспериментальных результатов для широкого набора ионов и условий облучения; при этом значение $E_{b}$ оказалось близким к полученному в работе [27]. Что касается параметров $r_{1}$ и $r_{t}$, то они входят лишь в предэкспоненциальные множители, и их вариация в разумных пределах не оказывает на результаты расчета существенного влияния.

Полученные решения системы уравнений позволяют анализировать зависимости концентрации точечных дефектов от условий облучения в широком диапазоне последних. Следует, однако, иметь в виду, что диапазон концентраций, в котором справедлив использованный подход, ограничен как снизу, так и сверху. Ограничение снизу обусловлено тем, что данный подход предполагает квазиоднородное распределение дефектов в плоскостях, параллельных поверхности. Как уже говорилось, это условие нарушается при малых дозах и малых плотностях ионного тока, когда за характерное „время жизни“ индивидуальных каскадов смещения не происходит их существенного перекрытия, и „события“ в каждом каскаде развиваются независимо друг от друга. Оценки показали, что для приводимых далее примеров расчета указанное условие приближенно можно считать выполненным. Верхний предел применимости уравнений (1) связан с тем, что при некоторой концентрации точечных дефектов происходит спонтанный переход кремния в аморфное состояние [8,28]. Расчеты по нашей модели позволяют оценивать дозы аморфизации в зависимости от массы иона, температуры и плотности ионного тока. Анализ этих зависимостей является предметом отдельного исследования.

Основные закономерности процессов накопления вакансий $\left(C_{v}\right)$ и дивакансий $\left(C_{v v}\right)$ рассмотрим на примере облучения кремния ионами $\mathrm{Si}^{+}$с энергией 100 кэВ. Зависимости концентраций вакансий и дивакансий, накопленных к моменту прекращения имплантации в максимуме распределений при $T=295 \mathrm{~K}$, от плотности тока $j$ при разных дозах $\Phi$ представлены на рис. 1. (Здесь и далее ионы считались однократно заряженными). Для других ионов получены качественно аналогичные результаты.

Из рис. 1 следует, что концентрации вакансий $C_{v}$ в интервале значений $j=0.2-10$ мкА $/ \mathrm{cm}^{2}$ не зависят, либо слабо зависят от дозы, практически достигая насыщения уже при дозе $3 \cdot 10^{12} \mathrm{~cm}^{-2}$. С увеличением $j$ при одной и той же дозе величина $C_{v}$ монотонно растет, причем при больших дозах эта величина практически пропорциональна $j$ во всем исследованном интервале плотностей тока, а при малых дозах $\left(\sim 10^{12} \mathrm{~cm}^{-2}\right)$ в области больших значений $j$ зависимость $C_{v}$ от плотности тока является сублинейной.

Для оценки закономерностей изменения уровня разупорядочения при комнатной температуре наибольший интерес представляет поведение дивакансий. При фиксированных значениях $j$ в области больших доз $\Phi$ их концентрация растет пропорционально дозе. При малых дозах зависимость имеет более сложный вид: наблюдается участок замедления, который особенно сильно выражен при больших $j$. Зависимость $C_{v v}$ от плотности тока близка к линейной в области больших $\Phi$, становится сублинейной при меньших дозах и немонотонной при дальнейшем снижении дозы.

Характер зависимостей $C_{v}$ и $C_{v v}$ от дозы и плотности тока объясняется конкуренцией нескольких процессов, одни из которых линейны (генерация вакансий, их захват на ловушки), другие (диффузия и объединение вакансий) - нелинейны. Увеличение степени радиационного повреждения с ростом $j$ при одной и той же дозе обусловлено повышением мгновенной концентрации вакансий $C_{v}$ вследствие увеличения скорости их генерации. Так как скорость образования дивакансий зависит от $C_{v}$ квадратично, а скорость потери вакансий за счет захвата

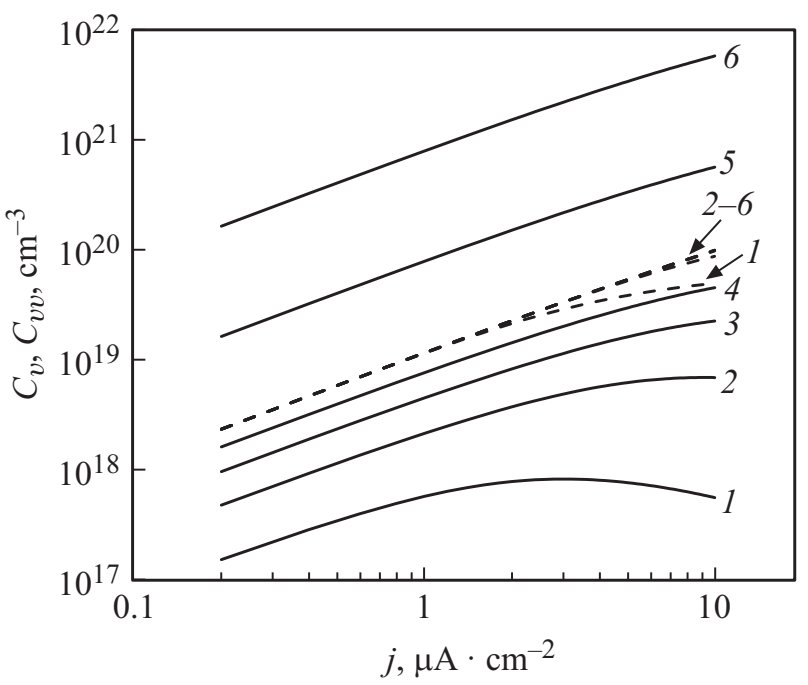

Рис. 1. Зависимости концентраций вакансий (штриховые линии) и дивакансий (сплошные линии) в максимумах концентрационных профилей от плотности ионного тока при $T=295 \mathrm{~K}$ для ионов $\mathrm{Si}^{+}$при $E=100$ кэВ, $\Phi, \mathrm{cm}^{-2}: 1-10^{12}, 2-3 \cdot 10^{12}$, $3-6 \cdot 10^{12}, 4-10^{13}, 5-10^{14}, 6-10^{15}$. 


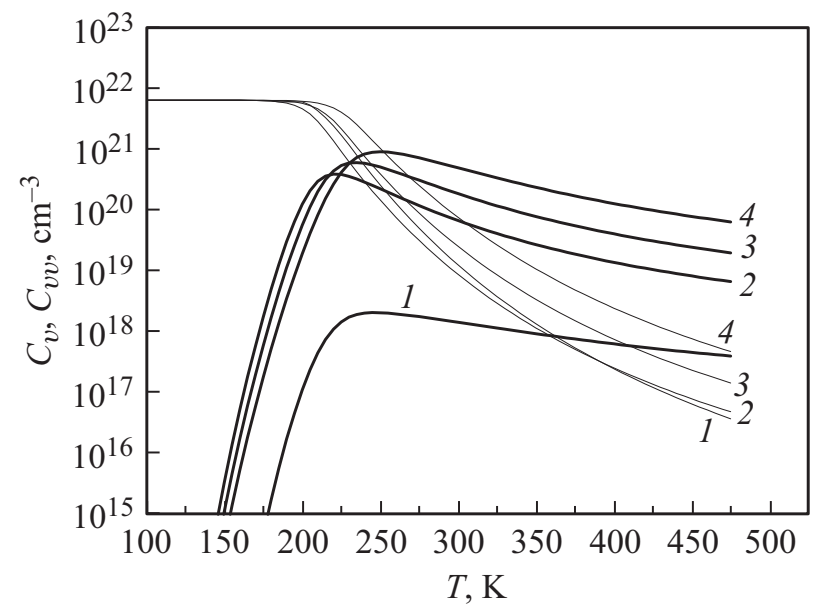

Рис. 2. Зависимости концентраций вакансий (тонкие линии) и дивакансий (толстые линии) в максимумах концентрационных профилей при облучении ионами $\mathrm{Si}^{+}(E=100$ кэВ, $\left.\Phi=10^{14} \mathrm{~cm}^{-2}\right)$ от температуры при облучении. Плотность ионного тока, мкА $/ \mathrm{cm}^{2}: 1-0.2,2-1,3-3,4-10$.

на ловушки пропорциональна первой степени $C_{v},-$ это при прочих равных условиях приводит к уменьшению потери вакансий с ростом $j$. Играет роль также соотношение между длительностью облучения (зависящей от $\Phi$ и $j$ ) и характерными временами квазихимических реакций: при малых плотностях тока и больших дозах время облучения относительно велико; за это время (при достаточно больших временах облучения - уже на начальной стадии облучения) успевает устанавливаться стационарная концентрация $C_{v}$, и скорость образования дивакансий становится постоянной. Но при малых значениях $\Phi$ и больших $j$ длительность облучения мала, так что концентрация вакансий не успевает достичь стационарного уровня; это приводит к замедлению роста и даже спаду $C_{v v}$ с увеличением $j$.

Таким образом, расчеты показывают, что полученные в работах $[2,3]$ в рамках аналитического приближения результаты справедливы при одних параметрах облучения и нарушаются при других параметрах. В частности, приблизительная пропорциональность между $C_{v v}$ и $j$ имеет место лишь в ограниченном интервале доз и плотностей ионного тока.

На рис. 2 представлены зависимости концентраций вакансий и дивакансий в максимумах распределений от температуры облучения ионами $\mathrm{Si}^{+}(100$ кэВ) для дозы $10^{14}$ при различных значениях $j$. Видно, что при всех значениях $j$ концентрация вакансий падает с ростом $T$. Это обусловлено ускорением процесса их потери за счет захвата ловушками и связывания в дивакансии, а также диффузионным расплыванием профилей распределения. Поведение дивакансий более сложное. Если при высоких температурах их концентрация уменьшается с ростом $T$, то при низких температурах она возрастает с увеличением температуры. Такая „аномалия“ обусловлена тем, что с понижением температуры снижается скорость связывания вакансий в дивакансии. При этом соотношение между концентрациями накопленных дивакансий и вакансий смещается в пользу последних. Поэтому уменьшение концентрации дивакансий с понижением температуры в области малых $T$ не означает снижения общей дефектности: преобладающим видом вакансионных дефектов становятся моновакансии.

Из рис. 2 можно видеть еще одну („аномальную“) закономерность: при низких температурах концентрация дивакансий снижается с ростом плотности ионного тока. Это тоже обусловлено низкой скоростью связывания вакансий в дивакансии, так что с увеличением $j$ (т.е. с уменьшением времени облучения при одной и той же дозе) все меньшее число вакансий успевает связаться в дивакансии.

Указанные закономерности для легких ионов носят общий характер, но при этом необходимо иметь в виду отмеченные выше ограничения области применимости нашей модели. Например, при достаточно больших массах ионов аморфизация в области низких температур может происходить при относительно небольших дозах, и „аномальное“ поведение дивакансий в этой температурной области не проявляется.

Представляет интерес вопрос о том, насколько сильно влияет на конечный результат диффузия вакансий, которая не учитывалась при аналитическом решении задачи в работах [2,3]. Выполненные расчеты привели к заключению, что учет диффузии при комнатной температуре приводит к значительному перераспределению точечных дефектов и при достаточно малых плотностях ионного тока существенно снижает концентрацию дефектов в области максимума распределения.

Приведенные зависимости относятся к моменту окончания облучения. Экспериментально степень повреждения чаще всего измеряется не во время облучения, а через некоторое время после облучения. Расчеты показали, что в рассмотренных интервалах доз и плотностей ионного тока пострадиционный период времени слабо влияет на концентрацию устойчивых при комнатной температуре дефектов по сравнению с периодом облучения.

\section{4. Заключение}

Использованная нами модель из работ [2,3] является упрощенной и не учитывает ряда особенностей радиационного повреждения. Так, процесс объединения вакансий в комплексы, скорее всего, не заканчивается на образовании дивакансий, а продолжается путем формирования более сложных комплексов. Кроме того, комплексы могут создаваться в каскадах смещения не только как вторичные, но и как первичные объекты. В качестве накапливающихся при облучении дефектов могут выступать, кроме вакансионных, и другие дефекты, например комплексы из межузельных атомов, устойчивые пары Френкеля [29], а также разупорядоченные области $[5,26]$. 
Выбор входных параметров, используемых в расчетах, не вполне однозначен. К тому же параметры, характеризующие процессы дефектообразования (например, энергетические барьеры элементарных актов с участием точечных дефектов, порог смещения $E_{d}$ ), вообще говоря, могут изменяться по мере накопления нарушений $[29,30]$.

Несмотря на указанные обстоятельства, предложенная в работах $[2,3]$ модель вторичного дефектообразования для легких ионов дает в целом правильное описание основных закономерностей кинетики накопления дефектов. Эту модель можно использовать для приближенных оценок степени разупорядочения кремния при ионном облучении легкими ионами, оценок доз аморфизации, а также для прогноза влияния условий облучения.

Достоинством разработанного нами комплекса программ является его гибкость, простота использования и нетребовательность к вычислительным ресурсам. Он позволяет оперативно анализировать влияние тех или иных изменений входных параметров и при необходимости включать в рассмотрение дополнительные процессы.

Исследование выполнено при поддержке РФФИ (грант № 17-02-01070) и частично Министерства образования и науки России в рамках государственного задания (№ 16.2737.2017/4.6).

\section{Список литературы}

[1] D.M. Paolillo. Nucl. Instr. Meth. Phys. Res. B, 365, 283 (2015).

[2] Н.П. Морозов, Д.И. Тетельбаум, П.В. Павлов, Е.И. Зорин. ФТП, 9 (12), 2292 (1975).

[3] N.P. Morozov, D.I. Tetelbaum. Phys. Status Solidi A, 37 (1), 57 (1976).

[4] А.И. Титов, А.Ю. Азаров, В.С. Беляков. ФТП, 37 (3), 358 (2003).

[5] K. Nordlund. Comput. Mater. Sci., 3, 448 (1995).

[6] F.G. Djurabekova, L. Malerba, C. Domain, C.S. Becquart. Nucl. Instr. Meth. Phys. Res. B, 255 (1), 47 (2007).

[7] L. Pelaz, L.A. Marques, J. Barbolla. J. Appl. Phys., 96 (11), 5947 (2004).

[8] A.I. Titov, G. Carter. Nucl. Instr. Meth. Phys. Res. B, 119, 491 (1996).

[9] O.W. Holland, S.J. Pennocook, L. Albert Gerald. Appl. Phys. Lett., 55 (24), 2503 (1989).

[10] T.R. Waite. Phys. Rev., 107 (2), 463 (1957).

[11] T.R. Waite. J. Chem. Phys., 28 (1), 103 (1958).

[12] N.P. Morozov, D.I. Tetelbaum. Phys. Status Solidi A, 51 (2), 629 (1979).

[13] J.F. Ziegler, M.D. Ziegler. Nucl. Instr. Meth. Phys. Res. B, 268 (11-12), 1818 (2010).

[14] А.Ф. Буренков, Ф.Ф. Комаров, М.А. Кумахов, М.М. Тёмкин. Пространственные распределения энергии, выделенной в каскаде атомных столкновений в твердых meлаx (М., Энергоатомиздат, 1985).

[15] W. Eckstein. Computer Simulation of Ion-Solid Interactions (Berlin, Springer Verlag, 1991).

[16] P. Sigmund. App. Phys. Lett., 14, 114 (1969).
[17] M.T. Robinson, I.M. Torrens. Phys. Rev. B, 9(12), 5008 (1974).

[18] S. Wolfram. „Mathematica“ A system for Doing Mathematics by Computer (Addison-Wesley Publishing Company, 1991).

[19] В.В. Емцев, Т.В. Машовец. Примеси и точечные дебекты в полупроводниках (М., Радио и связь, 1981).

[20] С.Н. Ершов, В.А. Пантелеев, С.Н. Нагорных, В.В. Черняховский. ФТТ, 19 (1), 322 (1977).

[21] E. Holmström, A. Kuronen, K. Nordlund. Phys. Rev. B, 78, 045202 (2008).

[22] V.L. Vinetski, T.R. Entinson, G.A. Kholodar. Phys. Status Solidi A, 67 (2), 477 (1981).

[23] K. Nordlund, A.E. Sand, F. Granberg, S.J. Zinkle, R. Stoller, R.S. Averback, T. Suzudo, L. Malerba, F. Banhart, W.J. Weber, F. Willaime, S. Dudarev, D. Simeone. Primary Radiation Damage in Materials (OECD, 2015).

[24] В.И. Окулич. Автореф. канд. дис. (Горький, ГГУ, 1976).

[25] В.К. Васильев, Н.П. Дикий, Е.П. Зорин, П.П. Матяш, П.В. Павлов, Н.А. Скакун, Д.И. Тетельбаум. ФТТ, 16 (4), 1064 (1974).

[26] Л.С. Смирнов. Физические процессы в облученных полупроводниках (Новосибирск, Наука, 1977).

[27] А.И. Баранов. ФТП, 18 (12), 2177 (1984).

[28] J.F. Gibbons. Proc. IEEE, 9, 1062 (1972).

[29] L. Pelaz, L.A. Marques, M. Aboy, J. Barbolla. Appl. Phys. Lett., 82 (13), 2038 (2003).

[30] Б.Л. Оксенгендлер, Н.Н Тураева, С.Е. Максимова, Ф.Г. Джурабекова. ЖЭТФ, 138 (3), 469 (2010).

Редактор А.Н. Смирнов

\section{The calculation of flux and temperature influence on damage accumulation kinetics at irradiation of Si with light ions}

\author{
E.V. Okulich ${ }^{1}$, V.I. Okulich ${ }^{2}$, D.I. Tetelbaum ${ }^{1}$ \\ ${ }^{1}$ Lobachevsky State University of Nizhny Novgorod, \\ 603950 Nizhny Novgorod, Russia \\ ${ }^{2}$ Nizhny Novgorod Institute of Management - \\ a branch of Russian Presidential Academy \\ of National Economy and Public Administration, \\ 603950 Nizhny Novgorod, Russia
}

Abstract The numerical calculations of the point defects (vacancies and divacancies) accumulation kinetics at irradiation of silicon by ions with masses $M_{1} \leq 31 \mathrm{amu}$. and energies $E \leq 100 \mathrm{keV}$ for different conditions of the radiation are produced. Previously suggested „diffusion-coagulation model“" was used, but without approximation of „weak diffusion“ which has been employed at analytic realization of this model. The main features of vacancies and divacancies concentrations vs. doses, ion current density and temperature under irradiation are analized. The physical interpretation of obtained results is carried out. The elaborated computing complex is rather flexible, permitting to analize the influence of input parameters variation and also to include (if required) additional processes into consideration. 